\title{
Effect of residual stresses on spallation of the film under impact by coated bullet
}

\author{
Chen-Wu Wu * \\ Institute of Mechanics, Chinese Academy of Sciences, Beijing, PR China 100190
}

\begin{abstract}
The dynamic equilibrium equation was derived for the film under the test of impact by coated bullet (ICB) to include the effect of the residual stress. Then, the finite element modeling was carried through to investigate the impact responses of the film of different initial stress states. The preliminary results revealed that the residual stresses will influence both the film stress and interface stress of the sample under the ICB test.
\end{abstract}

Keywords: Film; impact by coated bullet (ICB); residual stress; spallation; interface

\section{Introduction}

The mechanical reliability of film/ substrate system is largely dominated by the bonding strength between the film and substrate. In order to evaluate the interface strength of film and substrate, many kinds of methods, either quasi-static or dynamic loading have been developed and applied successfully [1]. Among the methods of dynamic loading, both the methods of laser spallation $[2,3]$ and impact by coated bullet (ICB) [4] utilize the reflected tensile stress of the input compressive stress pulse to make the separation of film off the substrate.

The method of Impact with Coated Bullet (ICB) has been justified experimentally to be able to evaluate the interface adhesion of metallic coating and substrate [5]. By impacting the substrate with a front-end-coated bullet, a compressive stress pulse of short duration is initiated to propagate toward the film under test. After transmitting through the interface of the sample, the input compressive pulse is reflected on the free surface of the film. The tensile stress will arise around the film and substrate

\footnotetext{
* Corresponding author: Institute of Mechanics, Chinese Academy of Sciences, No. 15 Beisihuanxi Road, Beijing, PR China 100190. Tel.: +86 10 82544271. E-mail:wcw_on@yahoo.com.cn; chenwuwu@imech.ac.cn.
} 
interface if the thickness of the bullet coat is appropriately chosen [4]. The propagation and evolution of the stress wave have been formulated for elastic system without considering the influences of the residual stresses [5, 6]. However, large residual stresses will inevitably be introduced into almost all kinds of films during deposition. These residual stresses shall contribute to the deformation of the surface layer and partly relax upon the separating of the film off the substrate, which will affect the fracture behaviors of the film and the film/ substrate interface. Such possible influences during the quasi-static loading experiments have drawn many attentions $[7,8]$. In the present research, the effects of residual stresses on the spalling fracture of the films under the test of ICB are investigated. First, the dynamic equilibrium equation of the film was derived to include the contribution of the residual stress. Then, the finite element model was set up to simulate the impact responses of the film of different initial stress states. Finally, the influences of the residual stresses on the film stress and interface stress of the sample under ICB test were analyzed.

\section{Theoretical description}

Ideally, the spallation of the film on the substrate subjected to impact by fronted-end-coated bullet (ICB) can be schematically depicted in Fig.1.

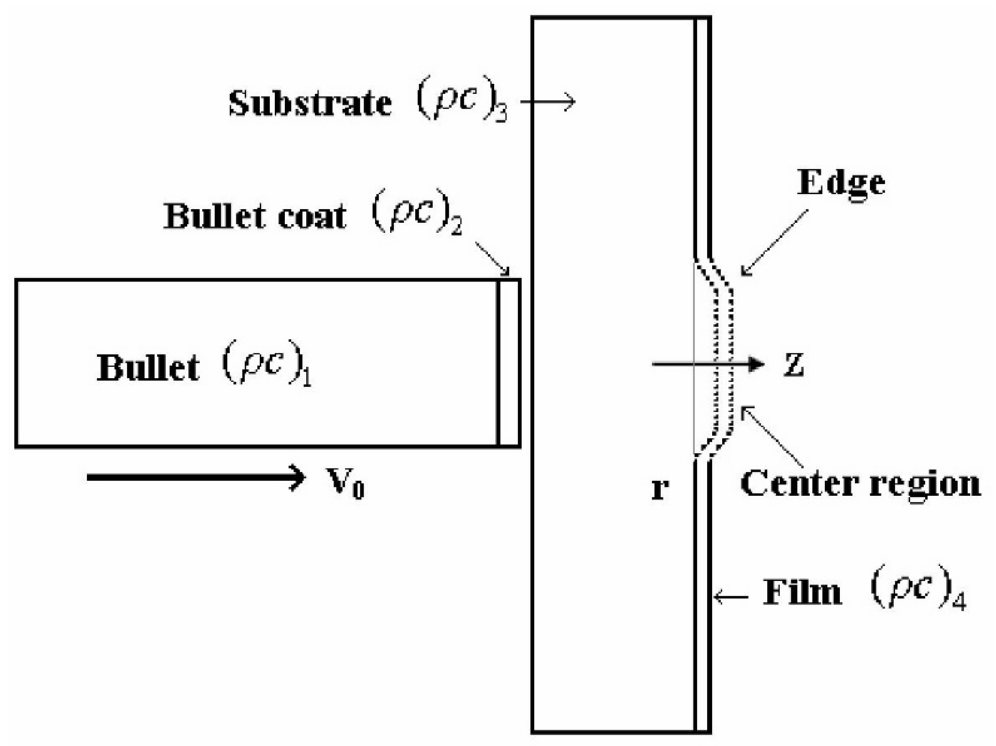

Fig.1 Sketch of spallation of the film under the ICB test 
The deformation of the sample can be approximately described by uniaxial strain wave theory within the center region covered by the bullet cross-section. In fact, the experimental results also reveal that the planar features of the deformation and interface fracture are obvious under ICB test, in particular where far enough away from the edge of the impact region [6]. Of course, the uniaxial strain model should not be directly applied to the deforming pattern around the circular edge of impact, because wherein there is great deformation gradient. In other words, the edge effect should not be ignored near the edge of the region covered by the impacting bullet.

As far as the edge effect is taken into account, the theory of thin plate bending may be adopted to describe the dynamic deformation of the film.

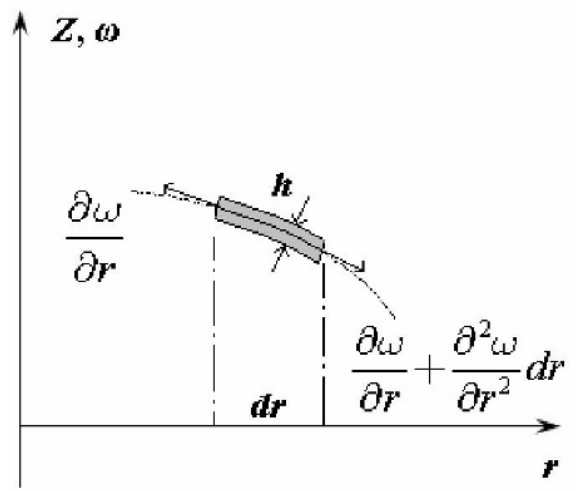

(a)

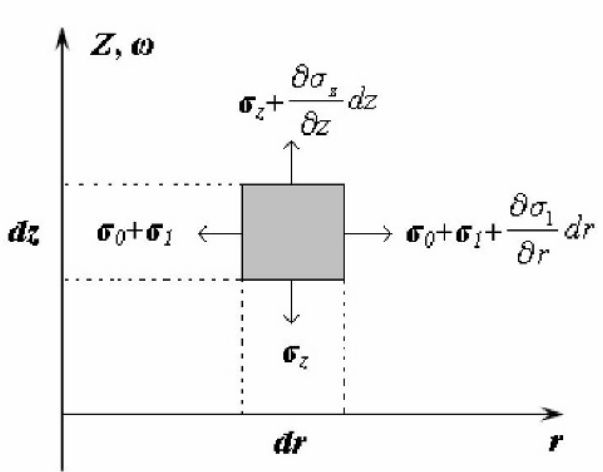

(b)

Fig.2 (a) Deformation of the infinitesimal section of total thickness and (b) Normal stresses acting on the infinitesimal ring of infinitesimal section

The deformation of a representative ring of infinitesimal length $d r$ and height $h$ of the film is illustrated in Figure 2 (a), and the normal stresses acting at the representative ring of square cross-section of infinitesimal dimensions are presented in Figure 2 (b). By neglecting the shear stresses according to the theory of thin plates [9], the equilibrium equation for such representative ring can be written as

$$
\begin{aligned}
& \left(\sigma_{z}+\frac{\partial \sigma_{z}}{\partial z} d z\right)(2 \pi(r+d r / 2))-\left(\sigma_{z}\right)(2 \pi(r+d r / 2)) \\
& \left(\sigma_{0}+\sigma_{1}+\frac{\partial \sigma_{1}}{\partial r} d r\right)\left(\frac{\partial \omega}{\partial r}+\frac{\partial^{2} \omega}{\partial r^{2}} d r\right)(2 \pi(r+d r) d z)+ \\
& \left(\sigma_{0}+\sigma_{1}\right) \frac{\partial \omega}{\partial r}(2 \pi r d z)=\rho \frac{\partial^{2} \omega}{\partial t^{2}}(2 \pi(r+d r / 2) d r d z)
\end{aligned}
$$


With eliminating the cancelable terms and neglecting the infinitesimal items of high orders, one can obtain the following control equation for the dynamic equilibrium of the film.

$\rho \frac{\partial^{2} \omega}{\partial t^{2}}-\frac{\partial \sigma_{z}}{\partial z}+\left(\sigma_{0}+\sigma_{1}\right) \frac{\partial^{2} \omega}{\partial r^{2}}+\left(\sigma_{0}+\sigma_{1}\right) \frac{1}{r} \frac{\partial \omega}{\partial r}=0$

By expressing the relationship between the stress and strain as

$\sigma_{z}=\varepsilon_{z} \times \frac{d \sigma_{z}}{d \varepsilon_{z}}$

and adopting the simplified treatment that

$\varepsilon_{z}=\frac{\partial \omega}{\partial z}$

, the equilibrium equation (2) can be further written as

$\rho \frac{\partial^{2} \omega}{\partial t^{2}}-\frac{d \sigma_{z}}{d \varepsilon_{z}} \frac{\partial^{2} \omega}{\partial z^{2}}+\left(\sigma_{1}+\sigma_{0}\right)\left(\frac{\partial^{2} \omega}{\partial r^{2}}+\frac{1}{r} \frac{\partial \omega}{\partial r}\right)=0$

As aforementioned, the uniaxial strain state can be assumed for the film and substrate away from the edge. By neglecting the radial deformation gradient, the equation (5) will reduce to

$\rho \frac{\partial^{2} \omega}{\partial t^{2}}-\frac{d \sigma_{z}}{d \varepsilon_{z}} \frac{\partial^{2} \omega}{\partial z^{2}}=0$

, which is identical to the one-dimensional wave equation [10]

$\frac{\partial^{2} \omega}{\partial t^{2}}-C_{L}^{2} \frac{\partial^{2} \omega}{\partial z^{2}}=0$

where

$C_{L}=\sqrt{\frac{1}{\rho} \frac{d \sigma_{z}}{d \varepsilon_{z}}}$

represents the longitudinal wave velocity.

By assuming the linear elastic behaviors for the materials, the first peak tensile normal stress acting at the film/ substrate interface has been obtained as [6]:

$\sigma_{i t b}=T_{34} \times T_{23} \times\left[v_{0}(\rho c)_{1} / 2\right]$

when $r_{21}(\rho c)_{3}+(\rho c)_{2} \leq 0$ 
and

$\sigma_{i t b}=T_{34} \times T_{23} \times\left[v_{0}(\rho c)_{1} / 2\right] \times\left(1-T_{21} \times T_{23}\right)$

when $r_{21}(\rho c)_{3}+(\rho c)_{2}>0$

Where $r_{i j}=\left((\rho c)_{j}-(\rho c)_{i}\right) /\left((\rho c)_{j}+(\rho c)_{i}\right)$ and $T_{i j}=2(\rho c)_{j} /\left((\rho c)_{j}+(\rho c)_{i}\right)$.

It is indicated in (9) and (10) that the residual stress has no contribution to the interface normal stresses at the center region of the linear elastic system under ICB test. However, once the nonlinear behaviors of materials are taken into account, the wave velocity, in particular in the surface layer should be influenced by the residual stresses [10]. This will influence the stress acting at the film/ substrate interface even around the center region away from the edge.

The deformation around the impact edge can be described by the equation (5), in which the in-plane stresses $\sigma_{1}$ will change with the stretching of the film. Thus $\sigma_{1}$ can be represented by some function $f(\omega)$ because that the stretch of the film is directly dependent on the magnitude of the off-plane displacement $\omega$. Therefore the equation (5) can be further written as

$\rho \frac{\partial^{2} \omega}{\partial t^{2}}-\frac{d \sigma_{z}}{d \varepsilon_{z}} \frac{\partial^{2} \omega}{\partial z^{2}}+\left(f_{1}(\omega)+\sigma_{0}\right)\left(\frac{\partial^{2} \omega}{\partial r^{2}}+\frac{1}{r} \frac{\partial \omega}{\partial r}\right)=0$

, which includes complex nonlinearity. As a preliminary attempt, we can assume that the circumferential strain is zero, that is

$\varepsilon_{\varphi}=\frac{1}{E}\left(\sigma_{\varphi}-\nu \sigma_{1}\right)=0$

Where $\sigma_{\varphi}$ represents the hoop stress. Thus,

$\sigma_{\varphi}=\nu \sigma_{1}$

By defining the radial strain as

$\varepsilon_{r}=\left((d r)^{\prime}-d r\right) / d r$

which is related to the stresses by 
$\varepsilon_{r}=\frac{1}{E}\left(\sigma_{1}-\nu \sigma_{\varphi}\right)$

Approximately, one has

$$
(d r)^{\prime}=\sqrt{\left(d r \frac{\partial \omega}{\partial r}\right)^{2}+(d r)^{2}}=d r\left(\sqrt{\left(\frac{\partial \omega}{\partial r}\right)^{2}+1}\right)=d r\left(2 \frac{\partial \omega}{\partial r}+1\right)
$$

Therefore

$\varepsilon_{r}=\left((d r)^{\prime}-d r\right) / d r=2 \frac{\partial \omega}{\partial r}$

By substituting (13) and (17) into (15), one can obtain

$\sigma_{1}=\frac{E}{1-\nu^{2}} \varepsilon_{r}=\frac{2 E}{1-\nu^{2}} \frac{\partial \omega}{\partial r}$

Finally, the equation (11) can be written as

$\rho \frac{\partial^{2} \omega}{\partial t^{2}}-\frac{d \sigma_{z}}{d \varepsilon_{z}} \frac{\partial^{2} \omega}{\partial z^{2}}+\left(\frac{2 E}{1-\nu^{2}} \frac{\partial \omega}{\partial r}+\sigma_{0}\right)\left(\frac{\partial^{2} \omega}{\partial r^{2}}+\frac{1}{r} \frac{\partial \omega}{\partial r}\right)=0$

The partial differential equation (18) involves at least the nonlinear items of $\frac{2 E}{1-\nu^{2}} \frac{\partial \omega}{\partial r}\left(\frac{\partial^{2} \omega}{\partial r^{2}}+\frac{1}{r} \frac{\partial \omega}{\partial r}\right)$, which make the analytical solution is somewhat hard to attain. For the sake of convenience, we use the finite element program Ls-dyna to analyze the impact responses of the sample herein.

\section{Numerical model and computational results}

\subsection{Numerical model}

The axisymmetric geometry model and the sketches of the mesh are shown in Fig.3. In Fig.3, the $\mathrm{X}$-axis starts from the symmetric axis and is parallel to the interface. Perfect bonding are assumed for the film/ substrate interface, across which the continuity conditions can be interpreted as

$\mathrm{u}_{n}^{+}=\mathrm{u}_{n}^{-}, \sigma_{n}^{+}=\sigma_{n}^{-}$and $\sigma_{\tau}^{+}=-\sigma_{\tau}^{-}$

Where

Superscripts ' + ' and '-' denote the two sides across the interface, $u$ is the displacement, 
$\sigma_{n}$ is the interfacial normal stress,

$\sigma_{n}$ is the interfacial shear stress.

The symmetric displacement constraints are applied at the nodes on the symmetry axis as the only boundary condition. The initial velocity of the bullet is $300 \mathrm{~m} / \mathrm{s}$ in the computation.

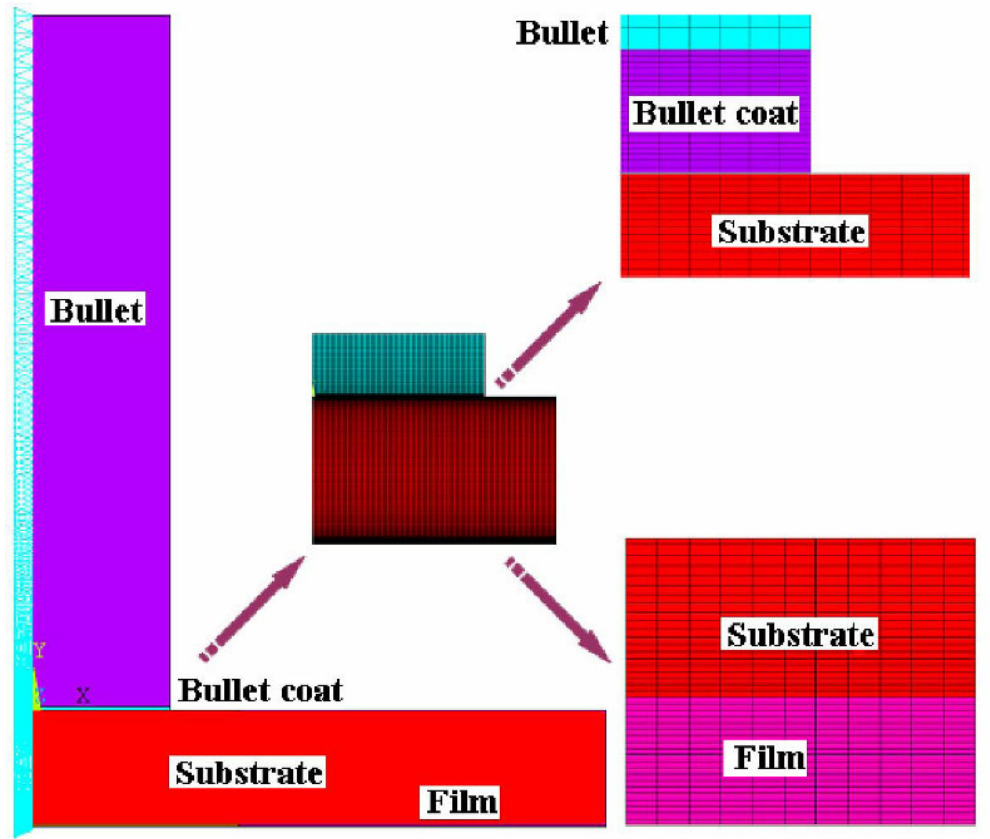

Fig.3 Finite element model of the bullet and sample

The rate-independent material model of bilinear isotropic plasticity is used in the computation, for which the main parameters are listed in Table1.

Table 1 Geometrical \& mechanical parameters

\begin{tabular}{cccccccc}
\hline & $t / \mathrm{mm}$ & $r / \mathrm{mm}$ & $\rho / \mathrm{kg} / \mathrm{m}^{3}$ & $E / \mathrm{GPa}$ & $v$ & $\sigma_{s} / \mathrm{MPa}$ & $E^{\prime} / \mathrm{MPa}$ \\
\hline Bullet & 30 & 6 & 1050 & 3.38 & 0.35 & 50 & 33.8 \\
Bullet coat & 0.1 & 6 & 7850 & 208 & 0.29 & 615 & 2080 \\
Substrate & 5 & 25 & 7850 & 208 & 0.29 & 900 & 2080 \\
Film & 0.1 & 25 & 8900 & 207 & 0.29 & 600 & 2070 \\
\hline
\end{tabular}

In table 1 ,

$t$ is the thickness of the bullet, bullet coat, film and the substrate,

$r$ is the radius,

$\rho$ is the density, 
$E$ is the elastic modulus,

$v$ is the Poisson's ratio,

$\sigma_{s}$ is the yield stress,

$E^{\prime}$ is the tangent modulus.

The technique of virtual thermal expansion is utilized to realize the action of the residual stress, of which the magnitude is $-1.73 \mathrm{GPa}$ in the initially compressed film and $1.73 \mathrm{GPa}$ in the initially tensioned film.

\subsection{Computational results}

The computation results reveal that the peak value of the first interface tensile pulse occurs at the moment of time $t=1 \mu \mathrm{s}$, therefore the deformation and stress distribution for the moment $t=1 \mu$ s are discussed largely.

The deformation of the initially stress-free film at the moment $t=1 \mu \mathrm{s}$ is shown in Fig. 4, in which the displacements are magnified by 50 times. It is revealed that the deformation is uniform and close to the uniaxial strain state within the center region under impact, while the off-plane displacement gradient arises obviously around the edge region.

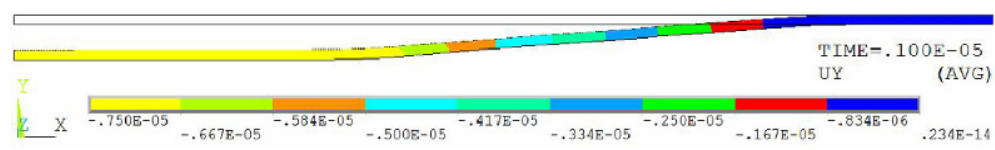

Fig.4 Deformation pattern at $t=1 \mu$ s of the initially stress-free film

The off-plane displacements of the film mapped onto the path parallel to the interface are plotted in Fig. 5 for the three different initial stress states.

In Fig. 5, the symbol

$\mathrm{rs}=0$ represents the initially stress-free state,

$\mathrm{rs}=-$ represents the initially compressed state,

$\mathrm{rs}=+$ represents the initially tensiled state,

$\mathrm{r} \_\mathrm{b}$ is the bullet radius.

The curves in Fig. 5 show that the residual compressive stress in the film will increase the off-plane displacements while residual tensile stress will decrease it. Moreover, it is indicated in Fig.5 that the off-plane displacements change in an almost linear law around the edge of the impact region. 


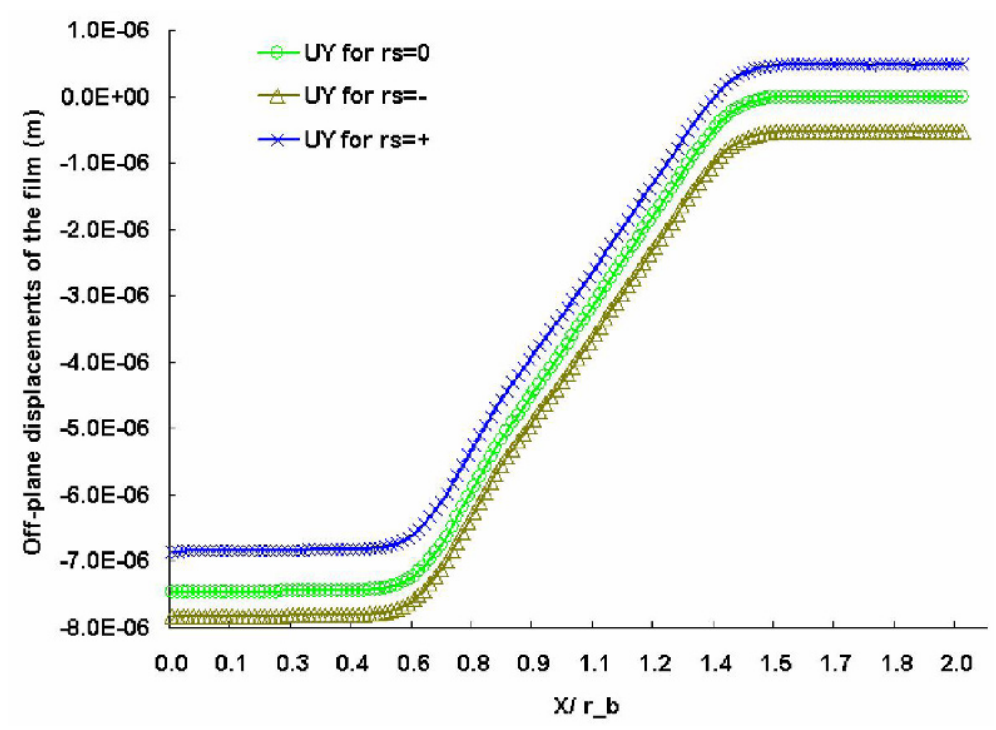

Fig. 5 Off-plane displacements at $t=1 \mu$ s of films of different initial stress states The contours of the radial stress component SX at time $t=1 \mu \mathrm{s}$ in the film of the three different initial stress states are further graphed in Fig. 6, in which the meanings of the symbols are identical to that in Fig. 5.

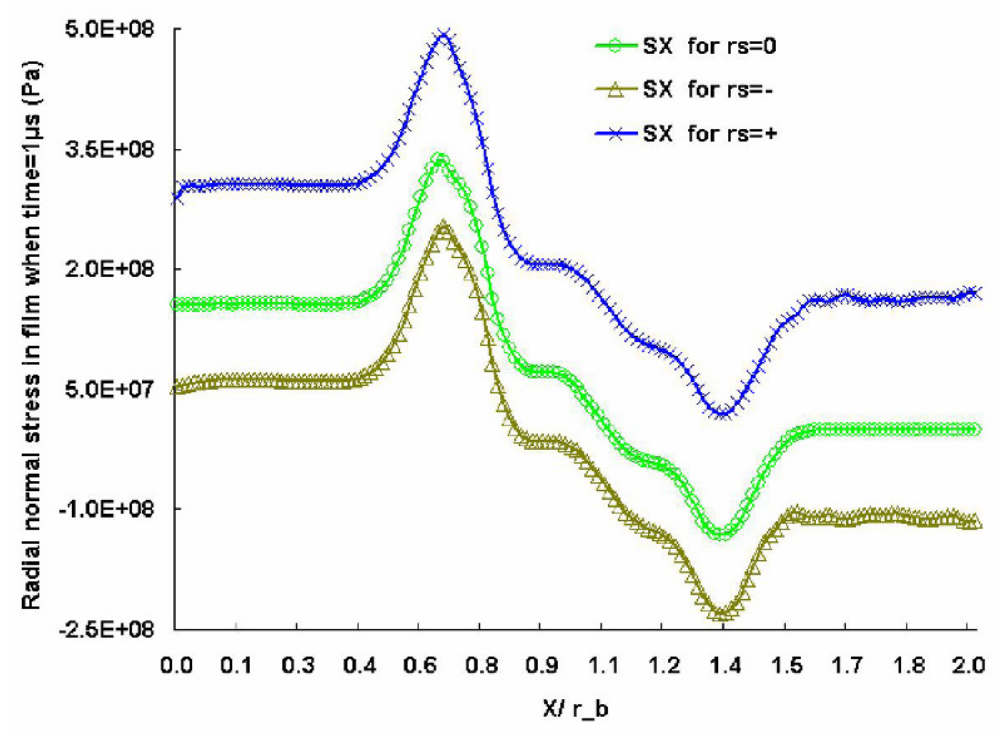

Fig.6 Radial normal stresses in the film at $t=1 \mu \mathrm{s}$

It is indicated in Fig. 6 that the film stress patterns are similar for all the three cases. Once again, the film stress is rather uniform within the center region under impact, while the obvious fluctuation arises around the edge regions as shown in Fig.6. It can also be noticed that in Fig. 6 that the maximum film stresses appear at the location about 0.7 times bullet radius distance from the center. Furthermore, just as one can 
expect, it is revealed in Fig. 6 that the residual compressive stress will decrease the stress of the film while residual tensile stress will increase it. This means that the residual tensile stress will promote the cracking of the film under impact, while the residual compressive stress will hold back the film cracking if neglecting temporarily the buckling of a greatly compressed film.

The free surface velocity histories of the three positions, i.e. the locations of No. 1 at the center, No. 2 half bullet radius distance from the center and No.3 a bullet radius distance from the center along the X-axis are plotted in Figs. 7 (a), (b) and (c). In Fig.7, the positive velocity values represent that in the direction of the outer normal vector of the surface.

By comparing the outcome graphs in Figs. 7 (a), (b) and (c), one can find out that the effects of the residual stresses on the surface velocity gradually become more obvious when the observed positions move from the center toward the edge. However, both the results on the three positions as illustrated in Figs. 7 (a) and (b) show that the first maximum and the first minimum values of the velocity are seldom influenced by the residual stress states. This means that the residual stress in the film may not contribute greatly to the spall velocity, especially when measured around the center region, which is usually used to describe the spallation strength of the test interface.

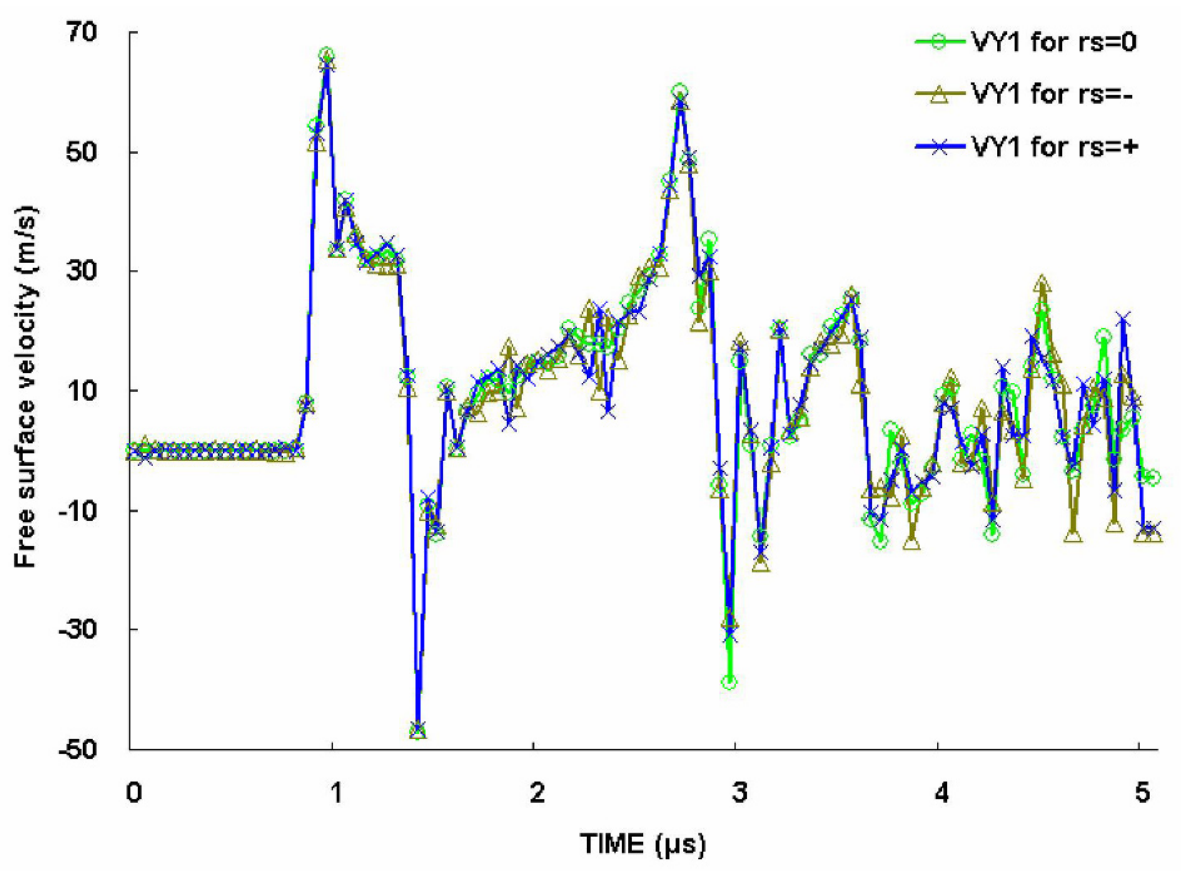

(a) 


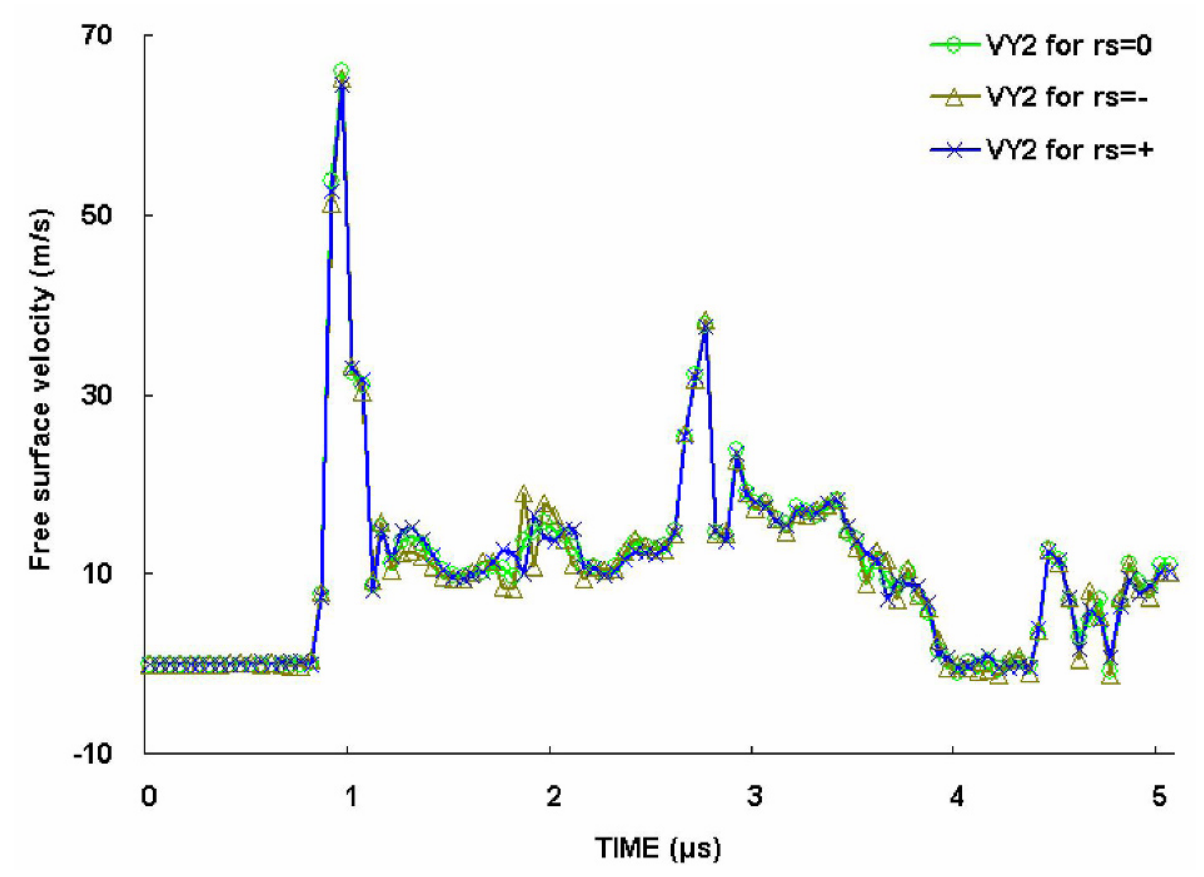

(b)

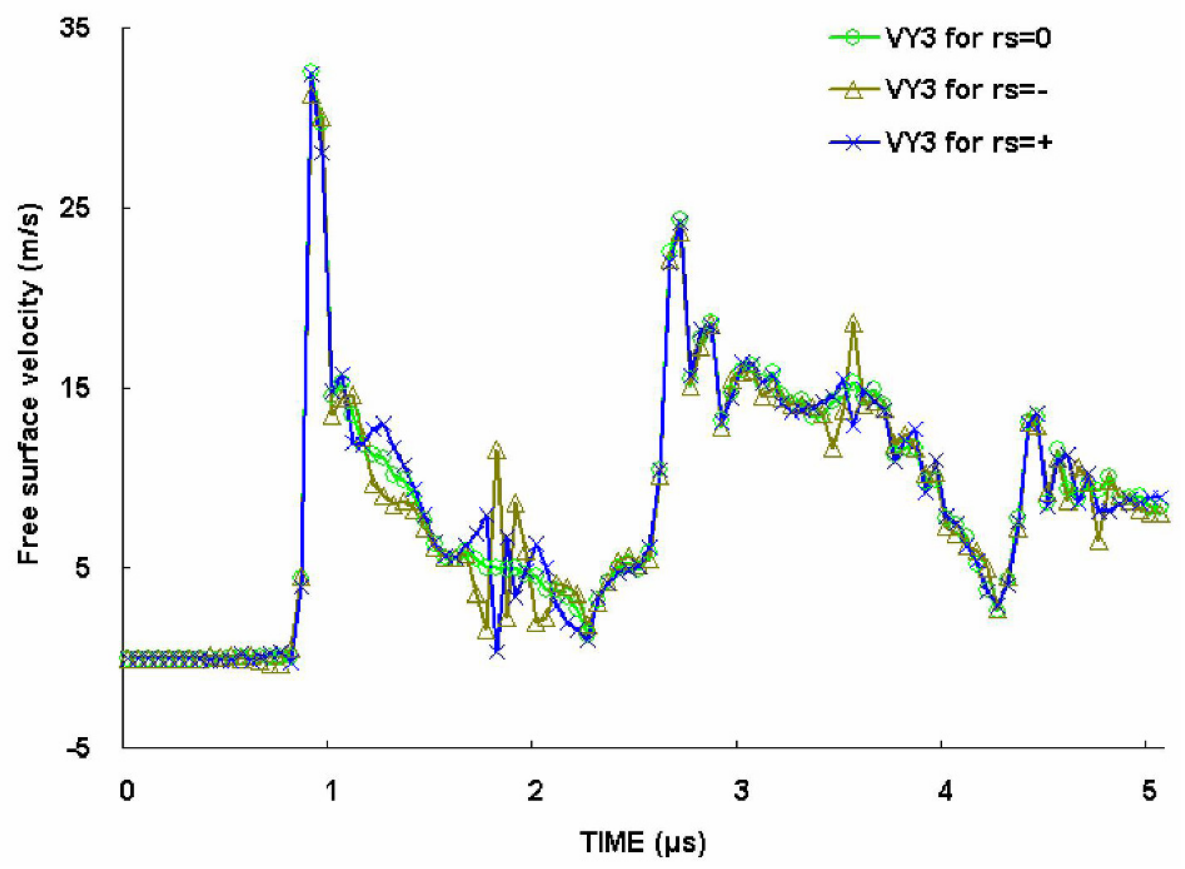

(c)

Fig.7 Surface velocity of the film at the (a) center, (b) half bullet radius and (c) a bullet radius distance from the center

The interfacial normal stress histories of the three positions, i.e. the locations of No. 1 at the center, No. 2 half bullet radius distance from the center and No.3 a bullet radius distance from the center along the $\mathrm{X}$-axis are graphed in Figs. 8 (a), (b) and (c). Once again, one can find out in Fig. 7 (a), (b) and (c) that the effects of the residual stresses 
on the interfacial normal stress become gradually greater when the studied positions move from the center toward the edge.

It is also shown in Fig. 7 that the maximum interfacial normal stress is obviously changed by the residual stress states.

The results displayed in Fig.8 (a) show that the residual compressive stress in the film will increase the overall maximum interfacial tensile stress at the center position.

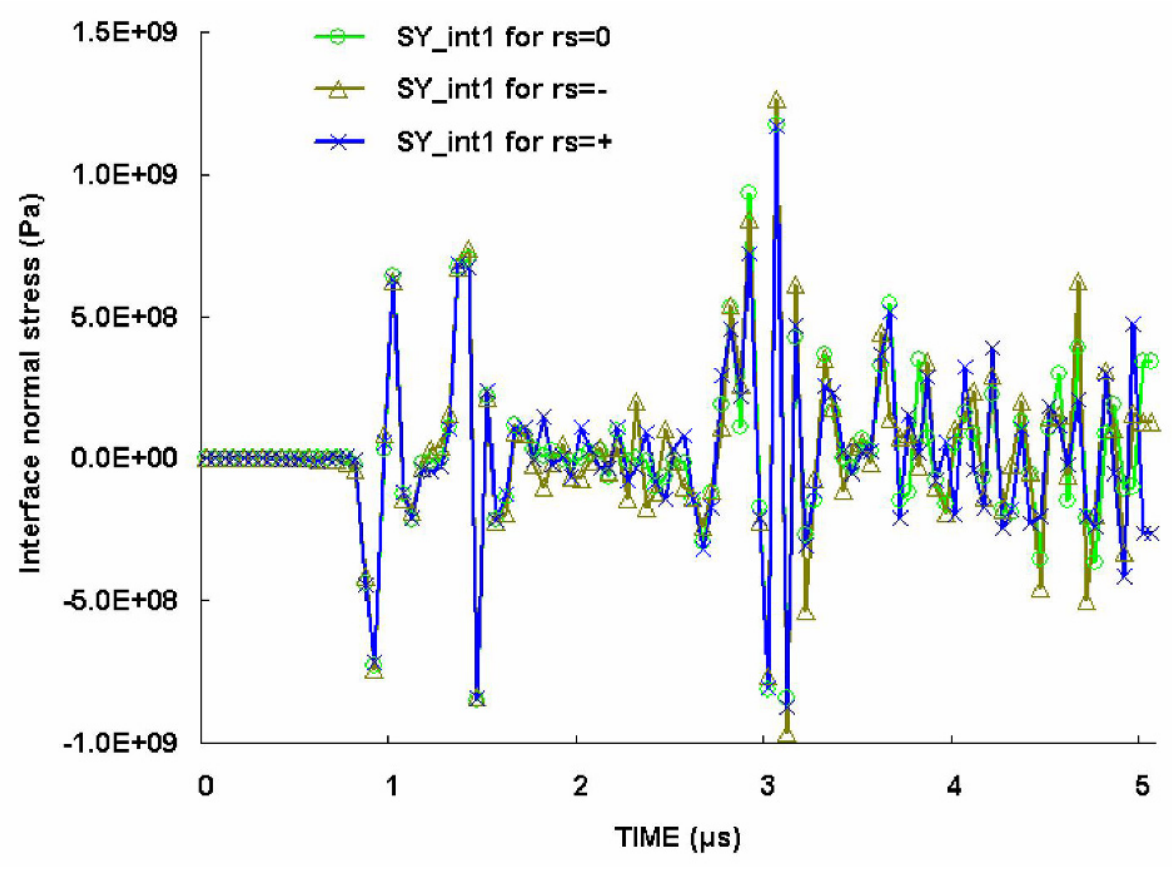

(a)

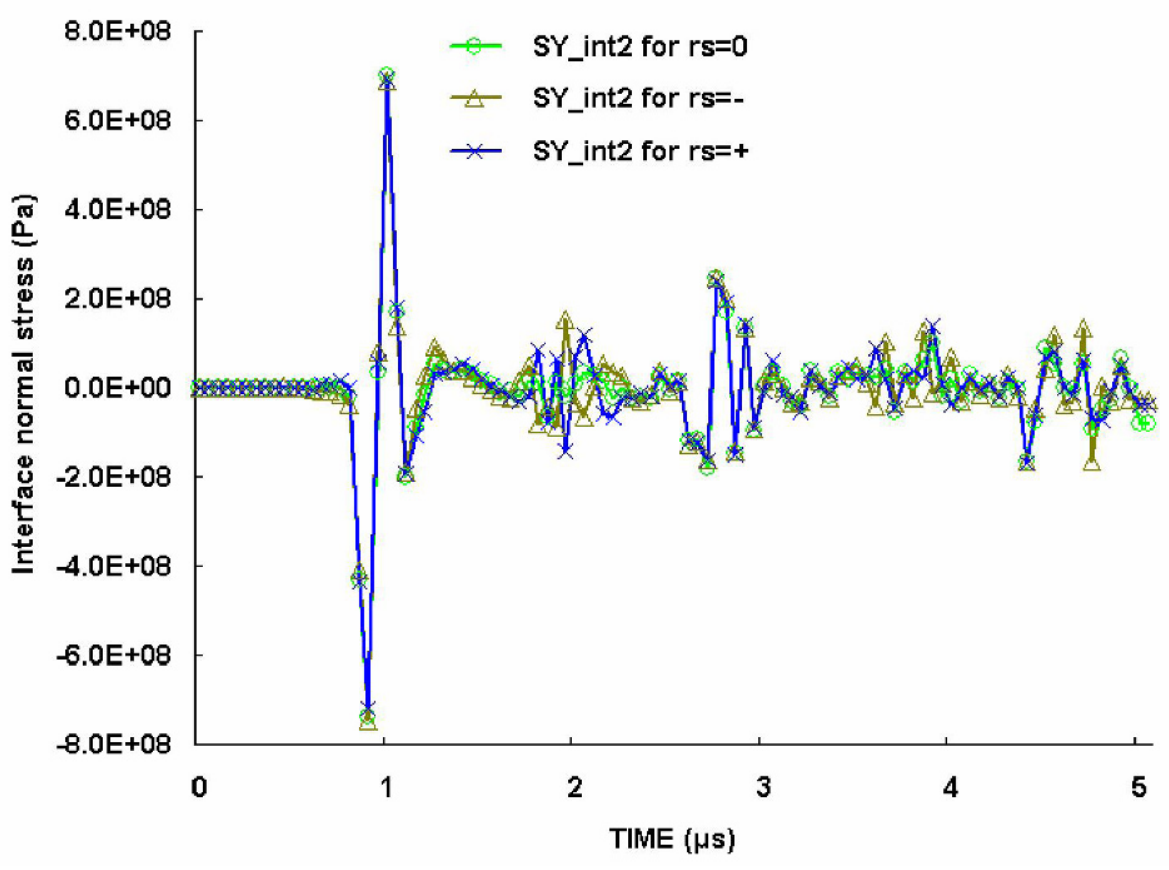

(b) 


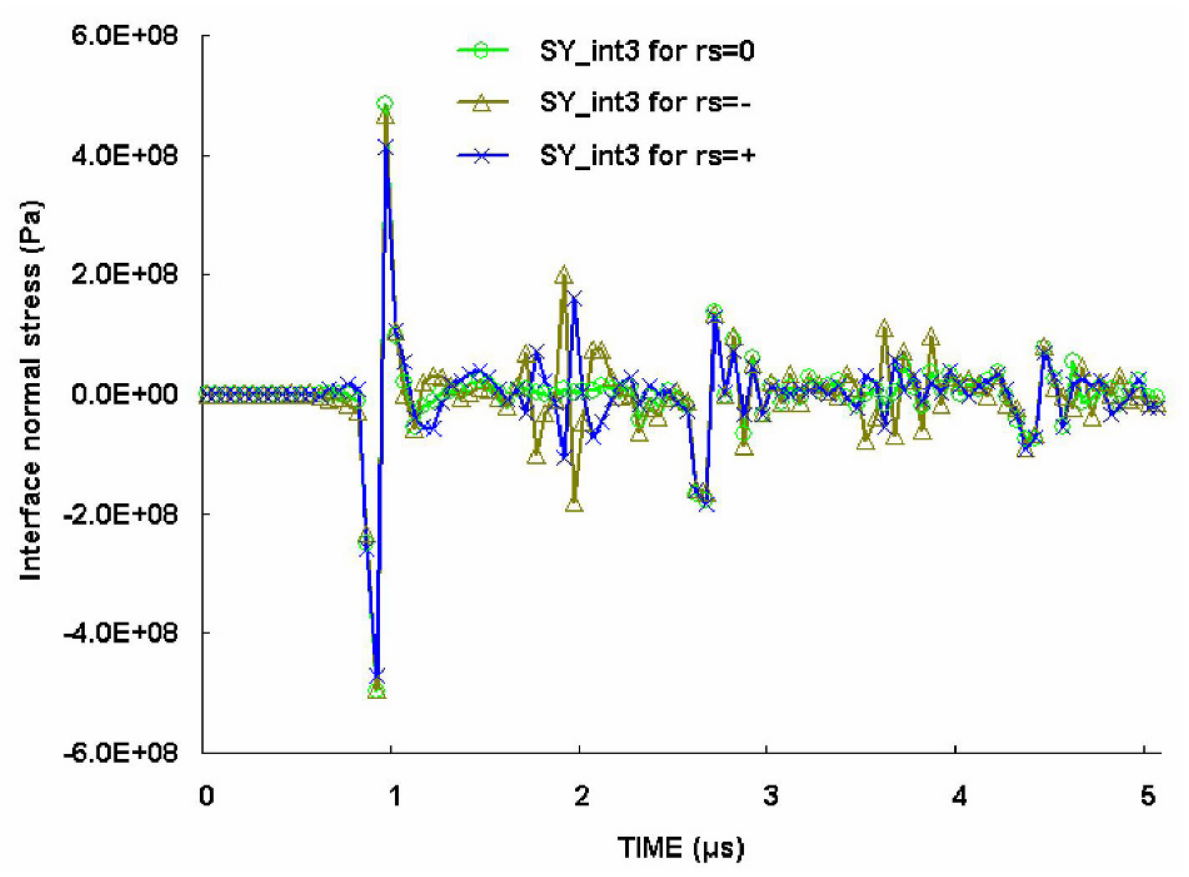

(c)

Fig. 8 Normal stress on the film/ substrate interface at the (a) center, (b) half bullet radius and (c) a bullet radius distance from the center

It is further indicated in Fig.8 (c) that both the residual compressive and residual tensile stress will reduce the first peak value of the interfacial tensile stress at the position of one bullet radius distance from the center.

The results graphed in Figs. 8 (b) and (c) show that both the residual compressive stress and tensile stress in the film will increase the subsequent peaks of the interfacial normal stress at the locations of half or one bullet radius distances away from the center.

\section{Conclusions}

The residual stresses influence greatly the off-plane deformation and the film stress of the sample under ICB test, in detail the residual compressive stress will partly relax the tension of the film while the residual tensile stress enhance it.

The effects of the residual stress on the surface velocity and the interfacial normal stress histories become more prominent when the observed positions move from the center to the edge. The peaks of the interfacial normal stresses are changed to a certain extent by the residual stresses. 


\section{Acknowledgements}

This work was supported by the National Natural Science Foundation of China (Grant No. 11002145).

\section{References:}

[1] R. Lacombe, Adhesion Measurement Methods: Theory and Practice, CRC Press, London, 2006.

[2] J.L. Vossen. Measurements of film-substrate bond strength by laser spallation. Adhesion Measurement of Thin Films, Thick Films and Bulk Coatings, American Society for Testing and Materials, ASTM STP 640 (1978):122-123.

[3] Junlan Wang, Nancy R. Sottos and Richard L. Weaver, Tensile and mixed-mode strength of a thin film-substrate interface under laser induced pulse loading, Journal of the Mechanics and Physics of Solid 52 (2004): 999-1022.

[4] Chen-Wu Wu, Zhi-Lin Wu, Kun Zhang and Guang-Nan Chen, Evaluation of film-substrate adhesion via impact using coated bullets, Journal of Mechanics of Materials and Structures, 10 (4) (2009) 1703-1709.

[5] Zhi-Lin Wu, Chen-Wu Wu, Guang-Nan Chen and Kun Zhang, On a novel method of impact by a front-end-coated bullet to evaluate the interface adhesion between film and substrate, Progress in Organic Coatings, 68 (1-2) (2010) 19-22.

[6] C.W. Wu, Z.L. Wu, C.G. Huang, Q. Zhang, L.R. He, K. Liu, G.N. Chen, Spallation of the thermal barrier coatings subjected to impact by coated bullet, submitted.

[7] Thouless, M.D.and Jensen, H.M., The effect of residual stresses on adhesion measurements, Journal of Adhesion Science and Technology 8 (6) (1994): 579-586(8).

[8] Pulin Nie, Yao Shen, QiuLong Chen and Xun Cai, Effects of residual stresses on interfacial adhesion measurement, Mechanics of Materials 41 (2009): 545-552.

[9] S. Timoshenko and S. Woinowsky-Krieger, Theory of Plates and Shells, 2nd ed., McGRAW-HILL Book Company, New York, 1987.

[10] Kolsky H., Stress Wave in Solids. Oxford: Clarendon Press, 1953. 